\title{
Hospital Care of Opioid-Exposed Newborns: Clinical and Psychosocial Challenges
}

\author{
Neera K Goyal, MD, MSc ${ }^{1,2 *}$, Jennifer McAllister, MD³
}

'Department of Pediatrics, Sidney Kimmel Medical College of Thomas Jefferson University, Philadelphia, Pennsylvania; ${ }^{2}$ Nemours/Al duPont Hospital for Children, Wilmington, Delaware; ${ }^{3}$ Department of Pediatrics, University of Cincinnati College of Medicine, Cincinnati, Ohio; ${ }^{4}$ Perinatal Institute, Cincinnati Children's Hospital Medical Center, Cincinnati, Ohio.

In the past two decades, the incidence of neonatal abstinence syndrome (NAS) has risen fivefold, mirroring the rise of opioid use disorder (OUD) among pregnant women. The resulting increases in length of stay and neonatal intensive care utilization are associated with higher hospital costs, particularly among Medicaidfinanced deliveries. Pregnant women with OUD require comprehensive medical and psychosocial evaluation and management; this combined with medication-assisted treatment is critical to optimize maternal and newborn outcomes. Multidisciplinary collaboration and standardized approaches to screening for intrauterine opioid exposure, evaluation and treatment of NAS, and discharge planning are important for clinical outcomes and may improve maternal experience of care. Journal of Hospital Medicine. 2020;15:613-618. (C) 2020 Society of Hospital Medicine $\mathrm{n}$ the past two decades, the incidence rate of opioid use disorder (OUD) among pregnant women has increased by more than $400 \%$, constituting a United States public health crisis.

Newborns exposed to intrauterine opioids are at risk for the postnatal withdrawal syndrome known as neonatal abstinence syndrome (NAS), which requires increased hospital resources, such as neonatal intensive care unit (NICU) admission and prolonged length of stay. ${ }^{2}$ Given the medical and psychosocial challenges associated with maternal OUD and NAS, a multidisciplinary, patient-centered approach to hospital care for affected newborns and their mothers is warranted. A large and growing body of research has focused on the epidemiology of NAS and approaches for its prevention, screening, and management. This review appraises updates to the literature within the past five years, with an emphasis on considerations for newborn discharge to promote optimal care for this population.

\section{DEFINITION}

NAS is a complex disorder arising from the abrupt cessation of placental transfer of opioids after birth, although other maternal substances, including benzodiazepines and other antidepressants, have been less commonly implicated. ${ }^{2}$ The term neonatal opioid withdrawal syndrome is sometimes used to indicate withdrawal from opioids specifically. ${ }^{3}$ The central and autonomic nervous systems and the gastrointestinal system (eg, tremors, increased muscle tone, high-pitched crying, feeding difficulties)

*Corresponding Author: Neera K. Goyal, MD, MSc; Email: neera.goyal@ nemours.org; Telephone: 215-861-8842.

Published online first February 20, 2020.

Received: July 31, 2019; Revised: December 5, 2019;

Accepted: December 6, 2019

๑ 2020 Society of Hospital Medicine DOI 10.12788/jhm.3369 are affected in NAS, with most newborns demonstrating symptoms within the first few days of life. ${ }^{4}$ Previously reported factors associated with NAS include opioid type, timing of exposure during pregnancy, maternal tobacco use, infant sex, and gestational age. ${ }^{5}$ Literature demonstrates that concurrent exposure to other prenatal substances, particularly antidepressants, benzodiazepines, and gabapentin, is significantly associated with increased risk of NAS. ${ }^{6}$ Recent studies also suggest that expression of NAS may relate to newborn genetic variations, particularly at the OPRM1, COMT, and CYP2B6 gene sites.7, 8

State health departments have increasingly deemed NAS as a reportable diagnosis for public health surveillance, which relies on the accurate diagnosis and documentation of NAS during birth hospitalization. ${ }^{9}$ The diagnosis codes for NAS include the International Classification of Diseases, Ninth Revision, Clinical Modification code (ICD-9-CM) 779.5 and the International Classification of Diseases, Tenth Revision, Clinical Modification ICD-10-CM code P96.1. ${ }^{10}$ However, given the variation in the presentation and severity of NAS, no consensus has been established with regard to a standardized case definition for reporting across hospitals and states. ${ }^{9}$ In fact, NAS should be conceptualized as a continuum of withdrawal symptoms along which every infant with intrauterine opioid exposure resides; this continuum ranges from minor findings, which do not affect the infant's ability to grow and develop, to severe withdrawal, resulting in excessive weight loss, dehydration, or seizures., ${ }^{3,11}$ Ultimately, the diagnosis of NAS is made clinically based on cardinal symptoms in the setting of known or highly suspected opioid exposure. In a recent study of Tennessee Medicaid claims data, $>25 \%$ of infants with a confirmed diagnosis code for NAS did not receive pharmacotherapy. ${ }^{10}$ Pharmacologic treatment of NAS, therefore, may be more appropriately considered as a marker of disease severity, rather than a requirement for diagnosis. 
TABLE 1. Common Opioids, Their Metabolites, and Approximate Urinary Detection Times ${ }^{34,35}$

\begin{tabular}{lll}
\hline Drug & Other metabolites & Urine detection time \\
\hline Heroin (diacetylmorphine) & 6 -AM & $2-8$ hours \\
\cline { 2 - 3 } Morphine & Morphine & $1-2$ days \\
\hline Hydromorphone & $(<3 \%)$ hydromorphone & $1-2$ days \\
\hline Fentanyl & Norfentanyl, despropionylfentanyl & $1-2$ days \\
\hline Codeine & Morphine, hydrocodone & $1-3$ days \\
\hline Hydrocodone & Hydromorphone, norhydrocodone, dihydrocodeine & $2-3$ days \\
\hline Oxycodone & Oxymorphone & $2-3$ days \\
\hline Tramadol & Noroxymorphone & $2-3$ days \\
\hline Buprenorphine & 0-desmethyltramadol & $2-4$ days \\
\hline Methadone & Norbuprenorphine & $1-7$ days \\
\hline
\end{tabular}

\section{EPIDEMIOLOGY}

Although the opioid crisis and resulting rise in NAS have affected communities across the US, substantial statewide variation exists, with extremes ranging from 0.7 per 1,000 births affected by NAS in Hawaii to 33.4 per 1,000 births in West Virginia. ${ }^{12}$ Within states, increased maternal OUD and NAS rates have also disproportionately affected rural communities, possibly due to reduced access to healthcare and mental health services and poor economic conditions..$^{13}$ A recent national study demonstrated that the proportion of newborns with NAS who were born in rural hospitals increased from $12.9 \%$ to $21.2 \%$ over the past decade; these rural newborns with NAS are more likely to be publicly insured and to require transfer after birth than newborns in urban hospitals. ${ }^{14}$ These data suggest a particular need among rural communities for increased resources targeting NAS care as well as maternal OUD prevention and treatment.

\section{RISK IDENTIFICATION}

The American College of Obstetricians and Gynecologists (ACOG) recommends early universal screening for maternal substance use at the first prenatal visit with a validated screening tool; examples include the 4Ps (parents, partners, past, and pregnancy), CRAFFT (car, relax, alone, forget, friends, trouble), and the National Institute on Drug Abuse quick screen, which have all been well studied and have a high sensitivity for detecting substance use and misuse. ${ }^{15}$

\section{Toxicology Screening}

Toxicology testing for both mother and newborn is helpful in identifying or confirming intrauterine exposures, particularly in cases of polysubstance use or when a newborn manifests signs of NAS but whose mother denies opioid use. All toxicology testing should be performed with the mother's consent, and any potential legal or mandatory ramifications of a positive test should be considered. Although universal maternal toxicology testing improves the identification of newborns at risk for NAS, this approach remains controversial; most hospitals use a riskbased approach for maternal toxicology testing. ${ }^{16,17}$

Newborn toxicology testing can be performed from samples of hair, urine, meconium, and umbilical cord. Although frequently used, newborn urine testing has the shortest window of detection, ie, the last few days prior to delivery (Table 1). Meconium drug testing has been considered the gold standard and can detect exposures from the last 20 weeks of gestation, providing information on chronic exposures. ${ }^{18}$ In a recent survey, $10 \%$ of hospitals reported using umbilical cord toxicology as the primary method for detecting intrauterine exposures..$^{17}$ This approach allows greater ease of specimen collection but may not yield results that are exactly equivalent to meconium testing. ${ }^{19}$

\section{MONITORING AND EVALUATION}

Close monitoring for development of NAS after birth is indicated for all newborns with confirmed or suspected intrauterine opioid exposure. Although the current American Academy of Pediatrics (AAP) guidelines recommend 3 days of newborn observation for exposure to short-acting opioids and 5 to 7 days for longer-acting opioids, substantial variation has been described across US hospitals in policies related to newborn length of stay for NAS observation. 17, 20 In most hospitals, monitoring for NAS occurs in the routine postpartum unit (ie, level 1 nursery), with transfer to the NICU if pharmacologic treatment is indicated. ${ }^{17}$

The most widely used assessment tool for NAS is the Finnegan Neonatal Abstinence Scoring System (FNASS) or the modified FNASS, which assigns points for the 21 most common opioid withdrawal symptoms based on perceived severity.17,21 
TABLE 2. Primary Pharmacologic Agents and Suggested Dosing and Weaning Regimens ${ }^{37}$

\begin{tabular}{|c|c|c|c|c|}
\hline & Administration route & Initiation dosing & Escalation & Weaning \\
\hline Morphine & Oral & $0.04 \mathrm{mg} / \mathrm{kg} /$ dose every 3 to 4 hours & Increase by $0.04 \mathrm{mg} / \mathrm{kg} / \mathrm{dose}$ if symptoms are not controlled & $\begin{array}{l}\text { Taper dose usually by } 10 \% \text { to } 20 \% \text { every } \\
2 \text { to } 7 \text { days }\end{array}$ \\
\hline Methadone & Oral, IV & 0.05 to $0.1 \mathrm{mg} / \mathrm{kg} / \mathrm{dose}$ every 6 hours & $\begin{array}{l}\text { Increase the dose by } 0.05 \mathrm{mg} / \mathrm{kg} / \mathrm{dose} \text { as needed, or increase } \\
\text { frequency to every } 4 \text { hours }\end{array}$ & $\begin{array}{l}\text { Taper dose by } 10 \%-20 \% \text { of therapeutic dose and/ } \\
\text { or extend dosing interval every } 1 \text { to } 2 \text { days as } \\
\text { tolerated }\end{array}$ \\
\hline Buprenorphine & Sublingual & $5.3 \mathrm{mcg} / \mathrm{kg} / \mathrm{dose}$ every 8 hours & $\begin{array}{l}\text { Increase dose in } 25 \% \text { increments if symptoms are not well } \\
\text { controlled; a rescue dose of } 50 \% \text { of the previous dose may } \\
\text { be used for between scheduled doses }\end{array}$ & Taper using $10 \%$ daily dose reductions \\
\hline
\end{tabular}

This tool allows for assessment of symptoms, helps determine need for pharmacologic intervention, and can guide monitoring of symptoms and weaning of therapy. A commonly used score cutoff of 8 is based on prior research validating scores $>8$ as indicative of withdrawal symptoms as opposed to normal newborn findings. ${ }^{22}$ Despite its popularity and widespread usage, FNASS has limitations, including the need for the newborn to be stimulated or disturbed to produce an accurate assessment and scoring for nonspecific signs of withdrawal, including sneezing, yawning, and stuffiness. Recent work has attempted to simplify and shorten the FNASS to elements that are unique and specific for withdrawal. ${ }^{23,24}$ Further research is needed to establish the validity of common scoring practices (ie, use of 8 as a cutoff) to determine the need for pharmacologic treatment. ${ }^{25}$

Recent studies suggest that simple, function-based assessments, such as the Eat, Sleep, Console (ESC) approach developed by Grossman and colleagues, may serve as an alternative to the FNASS for evaluating withdrawal. ${ }^{26,27}$ With ESC, the need for pharmacotherapy is evaluated by the newborn's ability to (1) eat (breastfeed successfully or eat at least $1 \mathrm{oz}$ per feed), (2) sleep uninterrupted for at least 1 hour, and (3) be consoled within 10 minutes. To date, research on the implementation of ESC has primarily focused on reducing length of stay and need for pharmacologic treatment in the context of quality improvement initiatives. ${ }^{26,27}$ Further prospective studies are warranted that compare ESC to traditional approaches involving the FNASS and that evaluate postdischarge outcomes including newborn weight gain, ongoing withdrawal symptoms at home, and readmission.

\section{NONPHARMACOLOGIC TREATMENT}

In recent years, research increasingly supports the critical role of nonpharmacological care in management of all opioidexposed newborns, regardless of NAS severity. ${ }^{11,27,28}$ Rooming-in of mothers or caregivers has been shown to decrease the need for pharmacologic treatment, shorten the length of stay, and reduce hospital costs. ${ }^{28,29}$ Other well-established practices include maintaining a low stimulus environment for infants with low lighting and sound, swaddling, maximizing caregiver contact with kangaroo care and skin to skin, and minimizing interventions. Therapeutic modalities, such as massage and music therapy, have been used for infants with NAS, but no evidence has supported their use. Recent studies have increasingly supported the use of acupuncture as an emerging modality in treating NAS. ${ }^{30}$

\section{Feeding}

Breastfeeding is encouraged for mothers who are stable on their methadone or buprenorphine maintenance treatment, are not using heroin or other illicit drugs, and have no other contraindications to breastfeeding, such as human immunodeficiency virus. ${ }^{31}$ Despite the known benefits of breastfeeding, which include decreased NAS severity, decreased need for pharmacological treatment, and shortened length of hospital stay, breastfeeding rates among mothers with OUD are low. ${ }^{31}$ Hospital policies that can promote maternal success in breastfeeding include tailored breastfeeding support, rooming in, and early, consistent maternal education on the benefits and safety of breastfeeding. ${ }^{32} \mathrm{~A}$ small percentage of hospitals use donor breastmilk for this population, although data on outcomes are limited. ${ }^{17}$ For formula-fed newborns, emerging research suggests that early initiation of high-calorie (22-24 kcal/ounce) formula may be beneficial to prevent excessive weight loss and poor weight gain after intrauterine opioid exposure..$^{33}$

\section{PHARMACOLOGIC TREATMENT}

When supportive therapy fails to adequately control symptoms of withdrawal, pharmacological management is initiated to improve infant discomfort, allow for adequate feeding and nutrition, and facilitate parental bonding (Table 2). ${ }^{11}$ Opioids are the primary agent used for pharmacologic treatment, and morphine is the most commonly utilized. ${ }^{17}$ Morphine is a short-acting opioid and can be prescribed either as a weightbased weaning protocol or a symptom-based regimen. Methadone is also widely used, and as a long-acting opioid, it has the advantage of twice daily dosing after the initial loading dose. Recently, buprenorphine, a partial mu opioid agonist with a long half-life, has emerged as a promising primary opioid treatment agent and has been shown to reduce the length of stay and the number of opioid treatment days compared with morphine and methadone. ${ }^{36}$

When the signs and symptoms of NAS are not effectively controlled with a primary opioid or in the case of polysubstance exposure, adjunctive agents are often used, with phe- 
TABLE 3. Adjunctive Pharmacologic Agents and Suggested Dosing and Weaning Regimens ${ }^{50}$

\begin{tabular}{|c|c|c|c|c|}
\hline & Administration route & Initiation dosing & Maintenance dosing & Weaning \\
\hline Phenobarbital & Oral, IV & (optional) 10 to $16 \mathrm{mg} / \mathrm{kg}$ once as loading dose & $\begin{array}{l}5 \mathrm{mg} / \mathrm{kg} / \text { day divided every } 12 \text { hours, beginning } \\
12-24 \text { hours after loading dose }\end{array}$ & $\begin{array}{l}\text { Decrease dose } 20 \% \text { every other day or such } \\
\text { that drug concentration decreases by } 10 \% \text { to } \\
20 \% \text { per day }\end{array}$ \\
\hline \multirow[t]{2}{*}{ Clonidine } & oral & 0.5 to $1 \mathrm{mcg} / \mathrm{kg} / \mathrm{dose}$ every 4 to 6 hours & $\begin{array}{l}\text { Increase over } 1 \text { to } 2 \text { days to maintenance dose } \\
\text { of } 3 \text { to } 5 \mathrm{mcg} / \mathrm{kg} / \mathrm{day} \text { in divided doses every } 4 \text { to } 6 \text { hours; } \\
\text { alternative maintenance dosing }\end{array}$ & $\begin{array}{l}\text { Wean as tolerated by } 25 \% \text { of the total daily } \\
\text { dose every other day }\end{array}$ \\
\hline & & & $\begin{array}{l}1.5 \text { to } 3 \mathrm{mcg} / \mathrm{kg} / \mathrm{dose} \text { every } 6 \text { hours as initiation/ } \\
\text { maintenance }\end{array}$ & \\
\hline
\end{tabular}

nobarbital and clonidine being the most common (Table 3). ${ }^{11}$ Regardless of opioid agent used, multicenter quality improvement initiatives demonstrate that having a standardized weaning protocol is critical to minimizing the overall length of stay and reducing the need for adjunctive agents. ${ }^{38,39}$ Additionally, modeling tools such as pharmacometrics for methadone and buprenorphine have shown promise in optimizing dose selection. ${ }^{40,41}$ Modeling may include pharmacodynamic data (ie, clinical response to treatment), pharmacokinetics (ie, measures of drug distribution and clearance), and other factors, such as patient demographics, intrauterine exposure type, and symptom severity. Future studies should examine weight versus symptom-based dosing regimens as well as compare weaning schedules versus "as needed" dosing regimens. ${ }^{11}$

\section{PSYCHOSOCIAL CONSIDERATIONS}

The need for comprehensive medical and psychosocial supports for mothers with OUD cannot be overstated, given the high rates of concurrent illicit or other substance use, comorbid depression and anxiety, physical and sexual trauma, poverty and homelessness, intravenous drug use, and sex-related risk patterns. ${ }^{15}$ Significant issues of healthcare-associated stigma and criminality also affect this population. As of 2019, 23 states and the District of Columbia classify substance use during pregnancy as child abuse under civil child-welfare statutes, potentially resulting in termination of parental rights. ${ }^{42}$ Studies of mothers with OUD have demonstrated that they often experience guilt, shame, and fear of loss of custody, all of which can impede their trust in hospital providers and future engagement in care. ${ }^{43}$ They also report frustration with and mistrust of NAS scoring assessments, which they can perceive to be disruptive and potentially biased. ${ }^{44}$ Multiple approaches should be considered to standardize and improve the hospital experience for this population, in a way that emphasizes the mother's role as a capable, respected participant in her newborn's care.

\section{Maternal Support}

A coordinated, multidisciplinary approach to comprehensively support mothers with OUD should involve team members from pediatrics, neonatology, obstetrics, nursing, social work, case management, and lactation..$^{35}$ This support includes screening for adequate resources and a safe, supportive, drug-free home environment as well as evaluating co-occurring mental health conditions. Referrals should be provided as needed to social services, postpartum psychiatry or behavioral health services, OUD treatment and relapse-prevention programs, and harm reduction services (eg, naloxone training). In addition to the healthcare team, other community members can be enlisted to serve as a trusted, consistent, and nonjudgmental support during the hospitalization; examples may include a peer support (another mother with OUD), an OUD program caseworker, or a doula. ${ }^{44}$

\section{Clinical Pathways}

Hospitals should establish clinical pathways for women with OUD to standardize care and communication across the continuum of care for themselves and their newborn, with input from all healthcare team members involved (prenatal, intrapartum, and postpartum). ${ }^{35}$ Early, consistent information should be provided regarding expected newborn hospital course, including toxicology testing, NAS monitoring, possible NICU admission, and involvement of social work.

\section{Provider Training}

Educational opportunities in the form of continuing medical education, in-service trainings, etc., should be provided for clinical staff who care for mothers with OUD and their newborns, regarding issues of substance use, stigma, bias, and traumainformed care. ${ }^{35}$ Online training resources are available through the American Society of Addiction Medicine, the ACOG, AAP, the Centers for Disease Control and Prevention, and the Substance Abuse and Mental Health Services Administration.

\section{DISCHARGE PLANNING}

Regardless of whether or not NAS is treated pharmacologically, newborns with opioid exposure may experience residual symptoms of withdrawal that persist for months. ${ }^{4}$ Current research suggests increased risk for morbidity, emergency department utilization, and rehospitalization after discharge in this population as well as difficulty in accessing and engaging with pediatric preventative care. 45,46

A clear plan should be established upon discharge to ensure optimal newborn care and follow-up. A complete record of the newborn's hospital stay, including maternal toxicology screenings and summary of any social work documentation, 
should be communicated to the primary care provider upon discharge. Close postdischarge monitoring involves addressing parenting knowledge gaps, assessing illness and injury risk, and evaluating for the presence of ongoing withdrawal symptoms. ${ }^{4}$ Primary care providers can also play a key role in assessing maternal stress, coping, and parenting skills as well as helping families connect to resources. Further research is warranted on how pediatric primary care systems can better build maternal trust, address parenting needs, and engage this population in routine well-child care. ${ }^{47}$

\section{Child Welfare, Early Intervention, and Other Services}

In general, newborn safety and keeping families intact should be prioritized, with disposition into foster care only in cases of concern for child maltreatment or neglect. Under the Child Abuse Prevention and Treatment Act (CAPTA), states are required to develop Plans of Safe Care for women and newborns affected by OUD, with the goal of fostering collaboration between healthcare and social service organizations around care of these families. ${ }^{48}$ Given the variable interpretation of Plans of Safe Care across the US, providers should be knowledgeable about state and local statutes and reporting requirements related to parental substance use.

As part of Plans of Safe Care, providers may be wellpositioned to initiate referrals for early intervention, home visiting, and other programs designed to provide developmental or wrap-around support for families. Under Part C of the Individuals with Disabilities Education Act, many states offer early intervention on the basis of NAS as an automatic qualifying diagnosis; however, attrition of eligible families along the referral and enrollment process is substantial. ${ }^{49} \mathrm{~A}$ standardized approach to discharging opioid-exposed newborns includes referrals to available resources and discussion of their importance with families and may increase utilization and decrease variation in care. ${ }^{50}$

\section{CONCLUSION}

Maternal OUD presents a unique combination of medical and psychosocial challenges that affect hospital care for mothers and their newborns. Optimal care for this population warrants a multidisciplinary team of providers who are knowledgeable, collaborative, and mindful of the important role of the mother as a key participant in her newborn's care. Despite a large and growing body of research focused on NAS prevention, screening, and treatment, ongoing efforts are needed to create hospital policies and clinical pathways that are responsive to the healthcare needs of this population, navigate sensitive issues of criminality and stigma, and ultimately support maternal parenting success.

Disclosures: The authors have no financial relationships and conflicts of interest relevant to this article to disclose.

Funding: Funding for this work was provided by Cincinnati Children's Hospital Medical Center and Nemours/Al duPont Hospital for Children.

\section{References}

1. Haight SC, Ko JY, Tong VT, Bohm MK, Callaghan WM. Opioid use disorder documented at delivery hospitalization - United States, 1999-2014. MMWR Morb Mortal Wkly Rep. 2018;67(31):845-849. https://doi.org/10.15585/mmwr. mm6731a1

2. Tolia VN, Patrick SW, Bennett MM, et al. Increasing incidence of the neonatal abstinence syndrome in U.S. neonatal ICUs. N Engl J Med. 2015;372(22):21182126. https://doi.org/10.1056/NEJMsa1500439

3. Devlin LA, Davis JM. A practical approach to neonatal opiate withdrawal syndrome. Am J Perinatol. 2018;35(4):324-330. https://doi. org/10.1055/s-0037-1608630

4. Kocherlakota P. Neonatal abstinence syndrome. Pediatrics. 2014; 134(2):e547-e561. https://doi.org/10.1542/peds.2013-3524

5. Kaltenbach K, Holbrook AM, Coyle MG, et al. Predicting treatment for neonatal abstinence syndrome in infants born to women maintained on opioid agonist medication. Addiction. 2012;107(Supplement 1):45-52. https://doi. org/10.1111/j.1360-0443.2012.04038.x

6. Huybrechts KF, Bateman BT, Desai RJ, et al. Risk of neonatal drug withdrawal after intrauterine co-exposure to opioids and psychotropic medications: cohort study. BMJ. 2017;358:j3326. https://doi.org/10.1136/bmj.j3326

7. Wachman EM, Hayes MJ, Brown MS, et al. Association of OPRM1 and COMT single-nucleotide polymorphisms with hospital length of stay and treatment of neonatal abstinence syndrome. JAMA. 2013;309(17):1821-1827. https:// doi.org/10.1001/jama.2013.3411

8. Mactier H, McLaughlin P, Gillis C, Osselton MD. Variations in infant CYP2B6 genotype associated with the need for pharmacological treatment for neonatal abstinence syndrome in infants of methadone-maintained opioid-dependent mothers. Am J Perinatol. 2017;34(9):918-921. https://doi. org/10.1055/s-0037-1600917

9. Jilani SM, Frey MT, Pepin D, et al. Evaluation of state-mandated reporting of neonatal abstinence syndrome - six states, 2013-2017. MMWR Morb Mortal Wkly Rep. 2019;68(1):6-10. https://doi.org/10.15585/mmwr.mm6801a2

10. Maalouf FI, Cooper WO, Stratton SM, et al. Positive predictive value of administrative data for neonatal abstinence syndrome. Pediatrics. 2019;143(1). https://doi.org/10.1542/peds.2017-4183

11. Mangat AK, Schmölzer GM, Kraft WK. Pharmacological and non-pharmacological treatments for the Neonatal Abstinence Syndrome (NAS). Semin Fetal Neonat Med. 2019;24(2):133-141. https://doi.org/10.1016/j.siny.2019.01.009

12. Ko JY, Wolicki S, Barfield WD, et al. CDC Grand Rounds: public health strategies to prevent neonatal abstinence syndrome. MMWR Morb Mortal Wkly Rep. 2017;66(9):242-245. https://doi.org/10.15585/mmwr.mm6609a2

13. Patrick SW, Faherty LJ, Dick AW, et al. Association among county-level economic factors, clinician supply, metropolitan or rural location, and neonatal abstinence syndrome. JAMA. 2019;321(4):385-393. https://doi.org/10.1001/ jama.2018.20851

14. Villapiano NL, Winkelman TN, Kozhimannil KB, Davis MM, Patrick SW. Rural and urban differences in neonatal abstinence syndrome and maternal opioid use, 2004 to 2013. JAMA Pediatr. 2017;171(2):194-196. https://doi. org/10.1001/jamapediatrics.2016.3750

15. Committee on Obstetric Practice American Society of Addiction Medicine. Committee Opinion No. 711: opioid use and opioid use disorder in pregnancy. Obstet Gynecol. 2017;130(2):e81-e94. https://doi.org/10.1097/ AOG.0000000000002235

16. Wexelblatt SL, Ward LP, Torok K, et al. Universal maternal drug testing in a high-prevalence region of prescription opiate abuse. J Pediatr. 2015;166(3):582-586. https://doi.org/10.1016/j.jpeds.2014.10.004

17. Bogen DL, Whalen BL, Kair LR, Vining M, King BA. Wide variation found in care of opioid-exposed newborns. Acad Pediatr. 2017;17(4):374-380. https:// doi.org/10.1016/j.acap.2016.10.003

18. Cotten SW. Drug testing in the neonate. Clin Lab Med. 2012;32(3):449-466. https://doi.org/10.1016/j.cll.2012.06.008

19. Colby JM, Adams BC, Morad A, Presley LD, Patrick SW. Umbilical cord tissue and meconium may not be equivalent for confirming in utero substance exposure. J Pediatr. 2019;205:277-280. https://doi.org/10.1016/j. jpeds.2018.09.046

20. Hudak ML, Tan RC, Committee on Drugs, Committee on Fetus and Newborn, American Academy of Pediatrics. Neonatal drug withdrawal. Pediatrics. 2012;129(2):e540-e560. https://doi.org/10.1542/peds.2011-3212

21. Finnegan LP, Connaughton JF, Jr, Kron RE, Emich JP. Neonatal abstinence syndrome: assessment and management. Addict Dis. 1975;2(1-2):141-158.

22. Zimmermann-Baer U, Nötzli U, Rentsch K, Bucher HU. Finnegan neonatal abstinence scoring system: normal values for first 3 days and weeks 5-6 in non-addicted infants. Addiction. 2010;105(3):524-528. https://doi. org/10.1111/j.1360-0443.2009.02802.x 
23. Jones HE, Seashore C, Johnson E, et al. Measurement of neonatal abstinence syndrome: evaluation of short forms. J Opioid Manag. 2016;12(1):1923. https://doi.org/10.5055/jom.2016.0308

24. Isemann BT, Stoeckle EC, Taleghani AA, Mueller EW. Early prediction too to identify the need for pharmacotherapy in infants at risk of neonatal abstinence syndrome. Pharmacotherapy. 2017;37(7):840-848. https://doi. org/10.1002/phar.1948

25. SchiffDM, Grossman MR. Beyond the Finnegan scoring system: novel assessment and diagnostic techniques for the opioid-exposed infant. Semin Fetal Neonat Med. 2019;24(2):115-120. https://doi.org/10.1016/j.siny.2019.01.003

26. Grossman MR, Berkwitt AK, Osborn RR, et al. An initiative to improve the quality of care of infants With neonatal abstinence syndrome. Pediatrics. 2017;139(6). https://doi.org/10.1542/peds.2016-3360

27. Wachman EM, Grossman M, Schiff DM, et al. Quality improvement initiative to improve inpatient outcomes for neonatal abstinence syndrome. J Perinatol. 2018;38(8):1114-1122. https://doi.org/10.1038/s41372-018-0109-8

28. Holmes AV, Atwood EC, Whalen B, et al. Rooming-in to treat neonatal abstinence syndrome: improved family-centered care at lower cost. Pediatrics. 2016;137(6). https://doi.org/10.1542/peds.2015-2929

29. MacMillan KDL, Rendon CP, Verma K, et al. Association of rooming-in With outcomes for neonatal abstinence syndrome: A systematic review and meta-analysis. JAMA Pediatr. 2018 Apr 1;172(4):345-351. https://doi. org/10.1001/jamapediatrics.2017.5195

30. Jackson HJ, Lopez C, Miller S, Engelhardt B. A scoping review of acupuncture as a potential intervention for neonatal abstinence syndrome. Med Acupunct. 2019;31(2):69-84. https://doi.org/10.1089/acu.2018.1323

31. Reece-Stremtan S, Marinelli KA. ABM clinical protocol \#21: guidelines for breastfeeding and substance use or substance use disorder, revised 2015. Breastfeed Med. 2015;10(3):135-141. https://doi.org/10.1089/bfm.2015.9992

32. Krans EE, Campopiano M, Cleveland LM, et al. National partnership for maternal safety: consensus bundle on obstetric care for women with opioid use disorder. Obstet Gynecol. 2019;134(2):365-375. https://doi.org/10.1097/ AOG.0000000000003381

33. Bogen DL, Hanusa BH, Baker R, Medoff-Cooper B, Cohlan B. Randomized clinical trial of standard- versus high-calorie formula for methadone-exposed infants: a feasibility study. Hosp Pediatr. 2018;8(1):7-14. https://doi. org/10.1542/hpeds.2017-0114

34. Lexicomp. Opioids, urine, screen and confirmation. Accessed September 4 2019. https://online.lexi.com/lco/action/doc/retrieve/docid/lthdph/382929

35. Mayo Clinic Laboratories. Opiates. Accessed September 4, 2019. https:// www.mayocliniclabs.com/test-info/drug-book/opiates.html

36. Kraft WK, Adeniyi-Jones SC, Chervoneva I, et al. Buprenorphine for the treat- ment of the neonatal abstinence syndrome. N Engl J Med. 2017;376(24):23412348. https://doi.org/10.1056/NEJMoa1614835

37. Lexicomp. Accessed September 4, 2019. https://online.lexi.com/lco/action/ home

38. Hall ES, Wexelblatt SL, Crowley M, et al. Implementation of a neonatal abstinence syndrome weaning protocol: A multicenter cohort study. Pediatrics. 2015;136(4):e803-e810. https://doi.org/10.1542/peds.2015-1141

39. Patrick SW, Schumacher RE, Horbar JD, et al. Improving care for neonatal abstinence syndrome. Pediatrics. 2016;137(5):38. https://doi.org/10.1542/ peds.2015-3835

40. Wiles JR, Isemann B, Mizuno T, et al. Pharmacokinetics of oral methadone in the treatment of neonatal abstinence syndrome: A pilot study. J Pediatr. 2015;167(6):1214-20.e3. https://doi.org/10.1016/j.jpeds.2015.08.032

41. Ng CM, Dombrowsky $\mathrm{E}$, Lin $\mathrm{H}$, et al. Population pharmacokinetic model of sublingual buprenorphine in neonatal abstinence syndrome. Pharmacotherapy. 2015;35(7):670-680. https://doi.org/10.1002/phar.1610

42. The Guttmacher Institute. Substance abuse during pregnancy. Updated November 1, 2019. Accessed November 20, 2019. https://www.guttmacher.org/ state-policy/explore/substance-use-during-pregnancy

43. Cleveland LM, Bonugli R. Experiences of mothers of infants with neonatal abstinence syndrome in the neonatal intensive care unit. J Obstet Gynecol Neonat Nurs. 2014;43(3):318-329. https://doi.org/10.1111/1552-6909.12306

44. Rockefeller K, Macken LC, Craig A. Trying to do what is best: a qualitative study of maternal-infant bonding and neonatal abstinence syndrome. Adv Neonat Care. 2019;19(5):E3-E15. https://doi.org/10.1097/ANC.0000000000000616

45. Liu G, Kong L, Leslie DL, Corr TE. A longitudinal healthcare use profile of children with a history of neonatal abstinence syndrome. J Pediatr. 2019;204:111117. https://doi.org/10.1016/j.jpeds.2018.08.032

46. Goyal NK, Rhode JF, Short V, et al. Well child care adherence during the first 2 years of life after intrauterine opioid exposure. Pediatrics. 2020;145(2):e20191275. https://doi.org/10.1542/peds.2019-1275

47. Short VL, Goyal NK, Chung EK, Hand DJ, Abatemarco DJ. Perceptions of pediatric primary care among mothers in treatment for opioid use disorder. J Commun Health. 2019;44(6):1127-1134. https://doi.org/10.1007/s10900-019-00701-1

48. Plans of Safe Care. Administration for Children and Families. Accessed September 1, 2019. https://www.acf.hhs.gov/sites/default/files/cb/pi1702.pdf

49. Peacock-Chambers E, Leyenaar JK, Foss S, et al. Early Intervention referral and enrollment among infants with neonatal abstinence syndrome. J Dev Behav Pediatr. 2019;40(6):441-450. https://doi.org/10.1097/DBP.0000000000000679

50. Crook TW, Munn EK, Scott TA, et al. Improving the discharge process for opioid-exposed neonates. Hosp Pediatr. 2019;9(8):643-648. https://doi. org/10.1542/hpeds.2019-0088 